Original Research Paper

\title{
One Can Slow Down the Aging through Antioxidants
}

\author{
${ }^{1}$ Raffaella Aversa, ${ }^{2}$ Relly Victoria V. Petrescu, ${ }^{1}$ Antonio Apicella and ${ }^{2}$ Florian Ion T. Petrescu \\ ${ }^{1}$ Advanced Material Lab, Department of Architecture and Industrial Design, \\ Second University of Naples, 81031 Aversa (CE), Italy \\ ${ }^{2}$ IFToMM, ARoTMM, Bucharest Polytechnic University, Bucharest (CE), Romania
}

Article history

Received: 26-11-2016

Revised: 27-11-2016

Accepted: 06-12-2016

Corresponding Author: Florian Ion T. Petrescu IFToMM, ARoTMM,

Bucharest Polytechnic

University, Bucharest (CE),

Romania

Email: petrescuflorian@yahoo.com

\begin{abstract}
Free radicals are natural nanoparticles which are doing their part well defined in the human cell, but then they must be annihilated or eliminated to not destroy the cell or another part of our body. It has been shown that residual free radicals in the body are responsible for the aging. The role to annihilate them is ful-filled by the antioxidants. Antioxidants are a group of compounds produced by the organism or that occur naturally in many foods. Antioxidants work together in the human body to maintain health and vigor, especially during the last decades of life. They do this by protecting us from harm caused by free radicals that can destroy skin and tissues healthy cells of the organism. Antioxidants are chemical compounds with many and varied applications. Naturally present in our diet, through fruits, vegetables, tea or red wine, they are recognized to be good for health. In fact, antioxidants protect our body's proteins and nucleic acids from oxidation. In the industry, antioxidants are also used as food additives to increase the shelf life of food. They are also present in the packaging or the wheels of cars because they prevent synthetic materials from becoming crumbly and brittle. Finally, in the cosmetics industry, creams containing antioxidants are known for their anti-aging properties. An antioxidant is a molecule that decreases or prevents the oxidation of other chemicals. Oxidation is part of an oxidation-reduction reaction that transfers electrons from a substance to an oxidizing agent. This reaction can produce radicals that cause destructive chain reactions. However, whatever the field of application, the unstable nature of antioxidants is a problem: The molecules degrade rapidly and have a particularly limited power of action over time.
\end{abstract}

Keywords: Biomaterials, Bioengineering, Biotechnologies, Antioxidants, Free Radicals, Slowing Aging, Skin Aging Slowing, Mitochondrion, Atp Molecule, Biochemical Processes, Predispose to Diseases, Oxidative Stress, Oxidation

\section{Introduction}

Antioxidants are chemical compounds with many and varied applications. Naturally present in our diet, through fruits, vegetables, tea or red wine, they are recognized to be good for health (McCall and Frei, 1999). In fact, antioxidants protect our body's proteins and nucleic acids from oxidation. In the industry, antioxidants are also used as food additives to increase the shelf life of food. They are also present in the packaging or the wheels of cars because they prevent synthetic materials from becoming crumbly and brittle. Finally, in the cosmetics industry, creams containing antioxidants are known for their anti-aging properties. An antioxidant is a molecule that decreases or prevents the oxidation of other chemicals. Oxidation is part of an oxidation-reduction reaction that transfers electrons from a substance to an oxidizing agent. This reaction can produce radicals that cause destructive chain reactions (Knight, 2000; Kowald and Kirkwood, 1996).

However, whatever the field of application, the unstable nature of antioxidants is a problem: The molecules degrade rapidly and have a particularly limited power of action over time. The research team of Yiannis Deligiannakis, a visiting professor at the Institute of Process Engineering at the Swiss Federal Institute of Technology in Zurich (ETHZ), has developed a nanoantioxidant with enhanced stability (Deligiannakis et al., 2012). This discovery is published in the journal ACS Applied Materials and Interfaces. 
An antioxidant is a molecule that decreases or prevents the oxidation of other chemicals. Oxidation is part of an oxidation-reduction reaction that transfers electrons from a substance to an oxidizing agent. This reaction can produce radicals that cause destructive chain reactions. Antioxidants are able to stop these chain reactions by reducing with the radicals and thus annihilating their action. These properties are found in the families of thiols and phenols (Alexeyev et al., 2004; Allen and Coombs, 1980; Ames, 1989).

Although oxidation reactions are necessary for life, they can also be destructive: Plants and animals use and produce many antioxidants to protect themselves, such as glutathione, vitamin $\mathrm{C}$ and vitamin $\mathrm{E}$, or enzymes such as Catalase, superoxide dismutase and certain peroxidases. A deficiency or absence of production of antioxidant enzymes leads to oxidative stress that can damage or destroy the cells. Similarly, our body is capable of producing, from the amino acid cysteine, a powerful antioxidant, $\alpha$-lipoic acid, also called lipoate.

Oxidative stress has been implicated in the pathogenesis of many human diseases, the use of antioxidants in pharmacology is therefore much studied to treat especially stroke and neurodegenerative diseases (Ahmed and Tollefsbol, 2001; Anisimov, 2001; Artandi, 2006; Austad, 1993; Beckman and Ames, 1998; Besedovsky and Del, 2008; Blackburn, 2000; Cadenas and Davies, 2000; Campisi, 1997; Cong et al., 1999; Daynes and Araneo, 1992; Dirks and Leeuwenburgh, 2006; Fabris, 1991; Finkel and Holbrook, 2000; Franceschi, 1989; Hayflick, 2004; Holliday, 2006; Huerre and Gounon, 1996; Kanungo, 1975; Wick et al., 1999).

However, it is not yet known whether oxidative stress is the cause or consequence of these diseases. Antioxidants are also important ingredients of dietary supplements in order to maintain health and prevent certain diseases, such as cancer or coronary heart disease. Although studies suggest that antioxidant supplements are beneficial to health, large clinical studies have not found any particular benefits and have even found that excess antioxidant supplements (or supplements) negative effects.

Antioxidants are also widely used by the industry as preservatives for food, cosmetics, or to preserve rubber or gasoline.

\section{History}

The term "antioxidant" (sometimes referred to as antioxidant) was originally used to refer to chemicals that prevent reactions with oxygen. In the late nineteenth and early twentieth centuries, the properties of antioxidants were widely studied for their use in industrial processes to reduce, for example, metal corrosion, vulcanization of rubber and polymerization of fuels in engines to explosion.
In biology, the first research on antioxidants concerned the reduction of the oxidation of unsaturated fatty acids, causing rancidity. Antioxidant activity was readily measured by enclosing fatty substances in hermetic containers with oxygen and then checking the absorption rate of the latter. However, it was only with the identification of vitamins $\mathrm{A}, \mathrm{C}$ and $\mathrm{E}$ that the importance of antioxidants appeared in the biochemistry of living organisms (Richter et al., 1988).

The possible mechanisms of antioxidants have been studied from the moment when it is understood that an antioxidant substance must itself be readily oxidizable. Research on the action of vitamin $\mathrm{E}$ in the limitation of lipid oxidation has demonstrated its role in the elimination of molecules containing an active oxygen atom before the latter attack the cells.

In 1956 Denham Harman published his hypothesis that free radicals $(\mathrm{RL})$ play a role in the aging process in humans (Harman, 2003; 1981; 1972; 1969; 1962; 1956; Viña et al., 2013).

Today, "The Free Radical Theory of Aging" is an integral part of the "passive aging" process, but there is as yet no absolute proof that free radicals are the cause of aging. A multitude of scientific studies shows, however, that free radicals are involved in the secondary aspects of diseases and changes in age. Today almost all of the scientific world believes that the balance between free radicals and antioxidants is involved in the prolongation of life expectancy but can also influence more complicated mechanisms and be involved in multiple pathologies and genetic diseases.

The first work on free radicals and human pathology was concentrated mainly on oxygen radicals. Indeed, after Harman's suggestion that free radicals were involved in human biochemistry, the first suspect of radical production was oxygen metabolism (Harman, 2003; 1998; 1972; 1969; 1962; 1956).

Oxygen radicals may form as a side reaction of ordinary metabolism of oxygen. About $97 \%$ of the oxygen used by the mitochondria to form water or carbon dioxide involves a step with two electrons. However, the remaining $3 \%$ of the oxygen used relates to secondary reactions relating to single electron transfers which can indirectly achieve the same result. Unfortunately, the oxygen radicals formed in $\propto$ process can also be involved in reactions that can cause significant damage to the body.

Indeed, stable molecules have even electrons, that is, electrons are in groups of two that share the same orbit to reach the lowest energy level for the number of electrons in the molecule. Free radicals are generally defined as molecules, molecular fragments or atoms that have one or more single (odd) electrons. In order to produce free radicals, the energy needed to break the doublet must be provided. This energy can come from 
light (photolysis), from ionizing radiation (radiolysis), from biochemical reactions (cell metabolism).

Typical oxygen radicals include hydroxyl, alkoxyl, peroxyl and semiquinone radicals, anion peroxide radical and hypochlorous acid. Nitric oxide is considered a nitrogen radical and the role of these radicals has expanded our understanding of the pathology of free radicals.

Later, it was recognized that other non-radical oxygen species such as singlet oxygen and hydrogen peroxide could be involved in similar pathologies. Therefore, we now consider these destructive oxygen components as Reactive Oxygen Species or Reactive Oxygen Species.

The excess of RL in the cell causes characteristic lesions: Peroxidation of membrane phospholipids (PUFA) both peri-cellular and intracellular (mitochondrial membranes, lysosomial membranes, etc.) alteration of the d Enzymes with a sulfydrile group, denaturation of the structure of collagen microfibrils (bone, among others as in osteoarthritis), hyaluronic acid, denaturation of DNA and RNA.

In living matter, the creation of $\mathrm{RL}$ is done by capturing hydrogen atom (electron plus proton) at the expense of neighboring organic molecules. There is a disruption of a covalent bond between one carbon atom and one hydrogen atom The Result is a Dehydrogenation Which Creates New Radicals which in Turn Capture Electrons, etc.

Radical reactions are therefore chain reactions which involve stages of initiation, propagation and arrest. This chain reaction stop occurs when two LRs react together or by precisely intervening cellular antioxidants.

An antioxidant is defined as a substance that has a small concentration relative to the concentration of an oxidizable substrate, delays or prevents oxidation of the substrate.

\section{Antioxidants Act as a Catalyst to Prevent Oxidative Damage}

Until recently, the focus has been on the identification and measurement of oxygen free radicals and on antioxidants or combinations of antioxidants that can reduce the damage caused by free radicals.

We now understand much better the pathology of radicals, insofar as we understand that other free radicals can play a role and that the balance between radicals and antioxidants can influence gene expression (both upstream and downstream).

The balance between oxidants (radicals and nonradicals) and antioxidants is a regulatory factor that can determine the expression of genes. By the activation of electrons in the network of antioxidants, signals are sent to transcription factors sensitive to reduction and oxidation, such as nuclear factor kappa B, AP1 and P53. These transcription factors act as "on/off" switches to control the expression of protective genes that repair damaged DNA, activate the immune system, stop the proliferation of damaged cells and induce cellular apoptosis (death).

The kappa B nuclear factor is important in the expression of many genes whose proteins play a role in activities such as apoptosis, B and $\mathrm{T}$ cell development, antiviral and bacterial responses, multiple responses to stress and Embryonic development. The products downstream of the nuclear factor kappa $B$ include inflammatory cytokines, tumor necrosis factor, nitric oxide synthase, cell adhesion molecules, leukocyte activation and viral activation.

Every week new results are published around the world confirming the importance of modulating the oxidative stress associated with an excess of free radicals and/or a lack of antioxidant defenses, in particular in the processes of aging and associated pathologies.

For example, an Italian study (Paolisso) showed that healthy centenarians had blood levels of antioxidant vitamins, $\mathrm{C}$ and $\mathrm{E}$, higher than a comparable population but did not exceed 100 years (Aged 70 to 99); (Paolisso et al., 1998).

It is also the accumulation of progressively less and less well-repaired damage, linked to the radical corrosion which takes a central place in the increase with the age of the frequency of the degenerative pathologies. Antioxidants can play a major role in their prevention and even their treatment.

Thus, in healthy subjects aged 65-94 whose memory and vocabulary are tested after elimination of statistically confounding factors, it is the circulating levels of vitamin $\mathrm{C}$ and beta-carotene that emerge as predictors of the best performance (La Fata et al., 2014; Kang et al., 2009).

Patients with various dementias or Alzheimer's disease have lower levels of antioxidant vitamins than controls (Foy et al., 1999). When 633 healthy subjects over the age of 65 are followed in the United States and are reevaluated on average 52 months later, 91 of them show signs of dementia. Among regular consumers of vitamin C or E, no case of dementia is recorded (Morris, 2009).

The immune functions of 88 elderly people are clinically improved by vitamin E supplementation and the authors recommend a systematic upward revision with age of intake of this antioxidant (Meydani and Blumberg, 1989). A study in 72-year-old women with coronary artery disease or major depression, obtained in 4 weeks with $200 \mathrm{mg}$ of vitamin $\mathrm{E}$ and $1 \mathrm{~g}$ of vitamin $\mathrm{C}$ per day, an optimization of the anti-infectious defenses and a reduction of circulating oxidized fats (De and Fuente, 2002; Tosato et al., 2007).

In the United States and India, the Reaver Dam Eye Study in 252 subjects aged 50 to 86 found $60 \%$ fewer cataracts in those with the highest blood levels of vitamins $C$ and $E$ (Ravindran et al., 2011). Treatment of 71 patients with severe macular degeneration at high risk of irreversible blindness with high doses of anti-oxidants 
(lutein esters, zeaxanthin and cryptoxanthin) halted the loss of vision as it continued in the placebo group (AgeRelated Macular Degeneration Study Group).

In Finland 7526 men are followed for 21 years. Nineteen of them develop insulin-dependent diabetes. The risk of diabetes is $88 \%$ lower in one third of the subjects who have the highest circulating rate of vitamin E adjusted to cholesterol (Knekt et al., 1988). In noninsulin-dependent diabetics with early neuropathy the administration of $900 \mathrm{mg}$ of vitamin $\mathrm{E}$ per day for 6 months results in an improvement in the statistically significant nerve conduction rate compared to the placebo group (Satoh et al., 2013; 2016).

Among 39,910 men and 86,245 women, all health professionals, followed by Harvard teams, consumers for more than 2 years of at least $100 \mathrm{IU}$ of vitamin E, 20 times the amount that is reached in the diet, Compared to non-users, a frequency of cardiovascular disease decreased from 37 to $41 \%$, respectively (Rimm et al., 1993) and the administration of even more vitamin $\mathrm{E}$ ( 400 or $800 \mathrm{IU}$ ) in more than 2000 coronary patients followed in Cambridge concludes a $77 \%$ decrease in the risk of non-fatal myocardial infarctions compared to the placebo group (Stephens, 1996).

All in all, whether in prevention or in therapeutics, the use of a complex combining natural vitamin E, vitamin $\mathrm{C}$, carotenoids and selenium is becoming more and more an obvious necessity. It remains to add dietary advice, avoidance of toxic, adaptation to stress and relevant physical and intellectual activities, as well as certain supplements specific according to the pathologies encountered.

\section{Materials and Methods}

Free radicals caused by pollution, unbalanced diet, tobacco, sun, excess sports or a stressful lifestyle can damage DNA and eventually lead to inflammation and cancers. Monitoring the proper balance between these free radicals and the body's defenses to neutralize them is therefore essential.

All living organisms use oxygen to metabolize and use dietary nutrients in order to produce energy for survival. Oxygen is thus an essential element to live. Oxygen meditates chemical reactions that metabolize fats, proteins and carbohydrates to produce energy.

\section{Black Side of Oxygen}

While oxygen is one of the most essential elements to live, it is also a two-edged sword. Oxygen is a highly reactive atom that is capable of part free radicals generally known as potentially damaging "molecules".

\section{Free Radicals}

These free radicals are capable of attacking the sound cells of the fuselage. This can result in damage, illness and severe disorders. Cell damage caused by free radicals appears to be an important contributor to aging and diseases such as:

\section{- Cancer}

- Cardiopathy

- Decline in brain function

- Decline in the immune system etc

In general, free radicals have been implicated in the pathogenesis of at least 50 diseases.

Since free radicals contain an odd electron they are unstable and reach out and capture electrons from other substances in order to neutralize. This initially stabilizes the free radical but produces of the others in the process. Soon a chain reaction begins and thousands of free radical reactions can occur in a few seconds on the primary reaction.

\section{Reactive Oxygen Species (ROS)}

ROS is a condition that surrounds all the highly reactive, oxygen-containing molecules, including free radicals. The types of ROS include the hydroxyl radical, hydrogen peroxide, the radical and nitric oxide radical of the superoxide anion, singlet oxygen, the hypochlorite radical and the various lipid peroxides. These can react with membrane lipids, nucleic acids, proteins and enzymes and other small molecules.

\section{Stress Oxidant}

Oxidative stress means an imbalance between prooxidants and antioxidant mechanisms. This results in excessive oxidative metabolism. This stress may be due to several environmental factors such as exposure to pollutants, alcohol, drugs, infections, poor diet, toxins, damage Oxidants from radiation therapy Etc. DNA, proteins and other macromolecules can result in a wide range of diseases especially human heart disease and cancer.

\section{Control of Free Radicals}

Normally the formation of free radical is regulated naturally by the various advantageous compounds known as antioxidants. When there is deficit damage these antioxidants due to free radicals can become cumulative and debilitating.

Antioxidants are able to stabilize, or put, free radicals before they off-attack cells.

\section{Antioxidants of Food}

There are several nutrients in food that contain antioxidants. Vitamin $\mathrm{C}$, vitamin $\mathrm{E}$ and beta-carotene are among the most commonly studied dietary antioxidants.

Vitamin $\mathrm{C}$ is the most important water soluble antioxidant in extracellular fluid. Vitamin $\mathrm{C}$ helps to 
neutralize ROS during water or water phase before it can attack lipids.

Vitamin E is the most important lipid soluble antioxidant. It is important as a network-breaking antioxidant in the cell membrane. It can protect membrane fatty acids from lipid peroxidation. Vitamin $\mathrm{C}$ plus is able to regenerate vitamin $\mathrm{E}$.

Beta-carotene and other carotenoids also have antioxidant properties. Carotenoids work in synergy with vitamin E.

\section{Antioxidant Deficits}

A low-fat diet can cause the deterioration of the absorption of beta-carotene and vitamin $\mathrm{E}$ and other fat-soluble nutrients. Fruits and vegetables are important sources of vitamin $\mathrm{C}$ and carotenoids. Whole grains and high quality vegetable oils are important sources of vitamin E.

Many central-derived substances are known as "phytonutrients," or "phytochemicals." These also possess antioxidant properties. Phenolic compounds such as flavonoids are such chemicals. These are found in several fruits, vegetables, green tea extracts etc.

\section{Antioxidants in the Human Body}

Regardless of the speed, the fuselage also has several antioxidant mechanisms that can protect against ROS assisted damage. The antioxidant enzymes-peroxidase, catalase and glutathione superoxide dismutase (GAZON) are such enzymes. They require micronutrient cofactors such as selenium, iron, copper, zinc and manganese for their activity. It has been suggested that an inadequate dietary intake of these trace minerals may also lead to low antioxidant activity.

To the saucepan or the crunch-to-salt, the antioxidants (vitamins, carotenoids, flavonoids ...) are the Samu of our cells. They boil to prevent, neutralize and/or repair the breakdown caused by free radicals generated by stress, pollution and junk food. Because they are the ones that accelerate aging by causing our cells to rust from the inside, favoring at the same time the appearance of diseases. Here's how to foil their traps with flavor.

In the family of carotenoids, red, orange, yellow and dark green plants are required for their vegetable vitamin A. They promote the rise of melanin, knowing that our internal production, which avoids us to roast, erodes from 10 to $20 \%$ every ten years.

We do not make fuss and eat them as much as possible with the skin, because the nutrients store just underneath to shield from external aggressions. Namely: The new carrot shoots, although more tender, contain less carotene than the big ones.

The right dosage: 4 apricots or $1 / 2$ mango or $1 / 2$ melon (the darkest possible) or $1 / 2$ red pepper per day.
The antioxidants survive hardly more than two days in the fridge, supply yourself in small quantities.

"Before the beach, we make a cure of exotic fruits (guava, mango, Passion fruit, kiwi, pomegranate). They concentrate the hard core of antioxidants: Vitamins A, C, E" highlights Serge Rafal, A doctor at the Tenon hospital in Paris. The reason? Fruits put the turbo to make these valuable antioxidants that protect the body from UV damage. Nevertheless, there is no Prolonged Orgy.

Translation: One does not drink 11 of mango or carrot juice per day non-stop, at the risk of overdosing one of the antioxidants, which would produce the opposite effect: To generate a deleterious oxidative stress. "The guarantee of balanced intake of antioxidants is to eat everything because the vitamins act in synergy and also neutralize this perverse effect," insists Professor Irene Margaritis, of the French Agency for Food Safety.

Plan of attack with lycopene (vegetal vitamin A). It preserves the density of the collagen fibers, support fabric anti-release and ensures the elasticity of the skin. Or? In raw tomato, juice or canned concentrate because, contrary to accepted ideas, it remains stuffed with lycopene in this form. Cooked with a fillet of olive oil, it is even better: The antioxidant is released in the heat and its action is boosted by the fat body. They are also found in guava, watermelon, pink grapefruit or apricot.

Vitamin A from meats, poultry, egg yolk, butter and offal is supplemented with retinol. These benefits are multiplied tenfold with vitamin $\mathrm{C}$ of strawberries, cabbage (darkest possible) or kiwifruit, because it is involved in the formation of collagen and it always arrives first in the blood plasma to trap free radicals.

Every day: 1 tray of $250 \mathrm{~g}$ strawberries or 1 slice of watermelon or 8 lychees or 1 citrus fruit or 2 kiwis. Add hazelnuts, almonds, wheat germ oil ( 1 tsp per day), grape seed or sunflower, rich in vitamin E, to perpetuate skin hydration and suppleness.

Flavonoids-which also fight free radicals-inhibit the action of an enzyme that promotes the appearance of cataract (opacification of the crystalline lens). We savor without moderation the violet, dark red, blue or black plants: Grapes, blueberries, blackcurrants, raspberries, plums...

To enhance the retina, we imitate Popeye: Spinach and sorrel are sources of zeaxanthin and lutein. Like corn and egg yolk.

The degradation of the nerve cells of the brain, linked to the oxidative stress, at the origin of the decline of the cognitive functions, is favored by a low consumption of the two carotenoids lycopene and zeaxanthin. Force on red, orange, yellow and dark green plants.

\section{Zinc and Selenium help to Circumscribe Inflammatory Processes and Promote Healing}

They are found in white fish, seafood and shellfish, in tuna and liver: To be consumed twice a week with rye bread, oats or spelled. 
Cherries, figs, grapes and berries (blueberries, blackberries, blackcurrants) are at the wedding: Their anthocyanins, polyphenols, are opposed to the formation of the atheromatous plaque, this accumulation of fat liable to obstruct the arteries and cause an infraction or a stroke. They reinforce, as a bonus, the wall of the capillary vessels.

Do not mash the garlic, it alone counts twelve antioxidants. Result: It fluidifies the blood and lowers the tension. Allegro forte on cinnamon, the most antioxidant spices: Consuming $1 \mathrm{~g}$ a day would cause cholesterol to fall by $25 \%$.

On the wine side, no excuses to celebrate outrageously the bandol under the plane trees, only the red is antioxidant ( 2 glasses maximum per day). Indeed, it is not the pulp of the grape that contains the antioxidants, but the skin. Notably resveratrol, a powerful polyphenol that the vines produce to protect themselves from rotting. Now the white wine does not require maceration between the juice and the skin and it is too short for the rosé.

Another solution: An alloy of grape marc and blackcurrant in the middle of a tablet of dark chocolate which contains, in the end, more antioxidants than two glasses of red wine. Chocolate New Tree "Eternity" in large surfaces.

Tea? Green, it is ten times richer in catechins (flavonoids) than black. Four or five cups a day lower blood sugar, reduce oxidative stress and prevent accumulation of abdominal fat, decreasing at the same time the risk of cardiovascular and cerebral diseases.

As the age of populations increases, the incidence of aging-related diseases also increases. Among them, cataracts and degenerative diseases such as macular degeneration of the eye (AMD) and Alzheimer's disease are the most commonly encountered. These pathologies generally induce irreversible lesions. The opacified crystalline lens is thus replaced by an artificial implant. On the other hand, Alzheimer's disease and macular degeneration of the eye are more difficult to treat.

Any factor affecting the progression or prophylaxis of these degenerative diseases has a significant socioeconomic impact and needs to be taken into consideration. Nutritional pathways are thus receiving increasing attention.

An elevation of oxidative stress due to excessive concentrations of radical species or deficiency of antioxidants seems to represent a significant risk factor for these pathologies. Limiting oxidative stress is thus the most promising current hypothesis in terms of prevention.

Micronutrients including vitamin $\mathrm{E}$, vitamin $\mathrm{C}$, vitamin $\mathrm{B}$ and certain carotenoids such as Lutein and zeaxanthin that make up our diet are likely to interfere with the mechanisms of prevention of these diseases.

Indeed, epidemiological studies of which the EVA study have demonstrated a significant correlation between a low status of these micronutrients and the cognitive decline of the elderly. The EVA study also showed an increase in oxidative stress in subjects with low carotenoids and vitamin $\mathrm{E}$.

The POLA study also showed a lower incidence of ocular diseases according to the biological status of vitamins E, C, B and certain carotenoids. Among the carotenoids, Lutein and zeaxanthin are of particular interest because they make up the macular pigment and are the only carotenoids present in the lens. Several studies demonstrate a preventive role of these carotenoids in cataract and AMD.

These observations are also supported by the results of fundamental studies on the prevention mechanisms involved. The antioxidants act either by neutralizing the reactive oxygen species or by preventing their propagation. Vitamin $\mathrm{C}$ acts in aqueous and hydrophobic media. Vitamin $\mathrm{E}$ is involved in membranes and lipoproteins. Once oxidized, these antioxidants are normally regenerated in their reduced form to be active again.

As aging is accompanied by less effective regenerative systems, it promotes the accumulation of cellular damage involved in degenerative pathologies. These effects are all the more important as the antioxidant status is low.

For the sake of prevention, it is therefore appropriate for healthy people to ensure optimal and adapted intake of micronutrients of vitamin E, C and carotenoids such as $\beta$-carotene, Lutein and zeaxanthin.

\section{Glutathione, the Master Antioxidant}

Few factors are as predictive of our life expectancy as the cellular levels of glutathione, dubbed the master antioxidant. According to Earl Mindell, author of What we should know about the super antioxidant miracle ("What we should know about the super antioxidant miracle"), without it we could literally not survive and other major antioxidants like vitamins $\mathrm{C}$ and $\mathrm{E}$ would be unable to do their job and effectively protect our body from disease. Over the years, levels of glutathione appear to be decreasing. Moreover, they are also reduced by a whole set of elements of our environment such as tobacco smoke, medications but also, the practice of endurance exercises, nutritional deficiencies or excessive alcohol consumption. It is possible to strengthen our body's glutathione levels with nutritional supplements such as glutathione itself but, most importantly, with substances such as alpha-lipoic acid, methionine, SAMe, or protein proteins.

Glutathione is a small protein composed of three amino acids: Cysteine, glutamic acid and glycine. It exists in a reduced form (GSH) and in an oxidized form (GSSG), both being in equilibrium with each other.

Glutathione is naturally present in virtually every cell in our body. No other antioxidant is as important as him. 
It is the regulator and regenerator of immune cells and the most effective detoxifying agent in the human body.

Without it, our cells would disintegrate under uncontrolled oxidation, our body would have little resistance to the attack of bacteria, viruses or cancer and our liver would dry out and die, an accumulation of toxins. Low levels of glutathione are associated with liver and immune dysfunction, heart disease, premature aging and even death.

\section{A Major Antioxidant}

Glutathione is the main water-soluble antioxidant of cytosol (the aqueous interior of cells) and participates directly in the destruction of reactive oxygen compounds. The two main antioxidant mechanisms of glutathione rely on its ability to detoxify hydrogen peroxide and other organoperoxidases as well as to defend the inside of the cells against oxidation by a redox phenomenon.

It is not only a powerful antioxidant but also plays a key role in the antioxidant network by recycling the oxidized forms of vitamin $\mathrm{C}$, thus restoring its antioxidant power.

\section{A Powerful Detoxifying Action}

Glutathione plays a major role in defending the body against pollutants. The liver is the organ most strongly involved in the detoxification of xenobiotics (substances foreign to the organism). It is also the most important storage center for glutathione that reaches its highest intracellular concentrations in healthy liver parenchymal cells (hepatocytes). Hepatocytes are highly specialized in the synthesis of glutathione from its precursors and the recycling of its oxidized form as well as in its use against potentially toxic substances.

Studies have shown that low levels of glutathione are synonymous with weakened liver functioning, resulting in increased amounts of toxins circulating in the body, damaging cells and organs.

Glutathione is involved in detoxification because it binds to toxins like heavy metals, solvents and pesticides and turns them into water-soluble compounds that can be easily eliminated in bile or urine.

\section{"Food" of the Immune System}

Glutathione is important for having a powerful immune system. By increasing its levels, it is possible to reverse the age-related collapse that occurs in immune function. High levels of glutathione allow our body to produce more white blood cells that are the first line of defense of the immune system. Dr. Gustavo Bounous, an eminent expert on glutathione, writes in this connection: "The limiting factor of an adapted activity of our lymphocytes (the white blood cells) is the availability of glutathione." In other words, one could write that glutathione is the "food" of the immune system.

\section{With Aging}

Our glutathione intake decreases with the years. The glutathione content of liver, kidney, heart and brain was $30,34,20$ and $30 \%$ lower in elderly mice (31 months), respectively, than in adult animals (17-23 months). Similarly, the concentrations of red blood cells in glutathione peroxidase are significantly lower in elderly than in young adults. Glutathione levels decrease substantially in the lens and red blood cells of the elderly. The decline in glutathione levels associated with aging is associated with an increase in diseases related to free radicals such as cancer, atherosclerosis or Alzheimer's disease.

\section{Glutathione and HIV}

Like other cell types, the proliferation, growth and differentiation of immune cells are dependent on glutathione. $\mathrm{T}$ and $\mathrm{B}$ lymphocytes require adequate levels of intracellular glutathione to differentiate and healthy subjects with relatively low levels of lymphocyte glutathione have a significantly lower CD4 count.

The decrease in experimentally induced glutathione levels inhibits the functioning of immune cells, indicating that intracellular glutathione status plays a central role in this domain.

In patients recently infected with HIV, glutathione levels in monocytes are abnormal, then normalized when the infection progresses at the same time as the GSH/GSSG ratio (reduced form/oxidized form) is disturbed. Plasma levels of cysteine and cystine, precursors of glutathione, are greatly decreased in HIVinfected patients.

Supplementation with $\mathrm{N}$-acetylcysteine helps patients infected with HIV, to restore normal levels of glutathione in the blood.

\section{Glutathione and Hepatic Impairments}

It was suggested that a decrease in glutathione levels was an important contributing factor to liver damage and that it reinforced the morbidity associated with liver malfunction. In a small study, abnormal plasma concentrations of glutathione were observed in patients with cirrhosis, regardless of their diet. A larger study demonstrated in 48 patients with cirrhosis that plasma glutathione levels were four to eight times lower than in healthy volunteers. A marked decrease in cysteine was also observed in severe cirrhosis.

\section{Glutathione and Neurodegenerative Diseases}

The brain is vulnerable to radical attacks. It is highly oxygenated, which makes it sensitive to the endogenous production of oxygenated radicals.

It also contains a high proportion of unsaturated fatty acids sensitive to peroxidation. Moreover, these regions 
of the brain rich in catecholamines are exceptionally fragile in the face of the generation of free radicals.

\section{Glutathione and Alzheimer's disease}

A study of the brain tissue of people who died as a result of Alzheimer's disease showed a lower content of glutathione and glutathione peroxidase as well as higher levels of peroxidized lipids than those of elderly people without significant pathology of the brain.

Alzheimer's disease has been linked to abnormally high levels of aluminum in the brain. When Alzheimer's disease patients are given metal chelators that push aluminum out of brain cells, their condition improves significantly and the progression of the disease is slowed. One of the most important functions of glutathione is to remove toxic metals such as aluminum, it appears that the age-related decline in glutathione levels could be the cause of an accumulation of toxic metals leading to Activity in the brain rich in oxygen. The latter leading to degenerative brain diseases such as Parkinson's disease or Alzheimer's disease.

\section{Glutathione and Parkinson's disease}

In Parkinson's disease, the loss of dopaminergic neurons results in increased dopamine metabolism increasing the formation of $\mathrm{H} 2 \mathrm{O} 2$ and resulting in the generation of strongly neurotoxic hydroxyl radicals. In the brain, the most important free radical neutralizer is glutathione. Its levels are low in patients with Parkinson's disease. Studies have shown that the loss of glutathione that manifests itself with aging in dopaminergic neurons can bring different alterations in the cells. These changes, combined with the oxidation of dopamine, seem to hasten cell death and advance the progression of Parkinson's disease.

A decrease in glutathione levels in brain tissue was related to the severity of the disabilities of patients who died of Parkinson's disease when no similar association was found with vitamin $\mathrm{C}$ levels.

\section{Glutathione and Eye Health}

Levels of lenticular glutathione in the eye have been shown to decrease with age, dropping from a concentration of $3.5 \mu \mathrm{mol} / \mathrm{g}$ at age 20 to $1.8 \mu \mathrm{mol} / \mathrm{g}$ to 65 years old.

Researchers have demonstrated the existence of a lack of glutathione in cataract lenses, indicating that it is a factor hastening the formation of senile cataract. Glutathione and its enzyme, glutathione peroxidase, act as powerful antioxidants to protect the transparency of the lens by stopping and/or repairing cell damage caused by free radicals. Experimental data show that taking a whey protein concentrate containing high concentrations of cysteine/cystine may provide the lens with a complement of glutathione and delay the development of cataract or even prevent its development.

\section{Increase Glutathione Levels}

Glutathione in its reduced form is not well assimilated by humans and it is advisable to administer large quantities of vitamins of the order of $500 \mathrm{mg}$ two to three times a day at least to increase serum levels of in a significant way. On the other hand, perlingual and acetylated forms are much better absorbed: 200 to 300 mg per day is sufficient to achieve the desired results.

\section{Cystine, a Preferred Precursor of Glutathione}

Glutathione is synthesized from L-glutamate, Lcysteine and L-glycine. The availability of L-cysteine may be considered as a limiting factor in the internal production of glutathione. The free amino acid cysteine is however not the best way to bring it to the cellular system. Potentially toxic, it is spontaneously destroyed in the gastrointestinal system and blood plasma. On the other hand, absorbed in the form of cystine (two molecules of cysteines joined by a disulfide bond), it travels safely through the gastrointestinal system and the blood plasma is rapidly reduced to two cysteine molecules at the entrance of the cells.

\section{$N$-acetylcysteine (NAC)}

Research, in vitro and in vivo, indicates that NAC is capable of stimulating the biosynthesis of glutathione. In vivo, NAC increases intracellular glutathione levels in erythrocytes as well as in liver and lung cells and restores the glutathione stock after they have been experimentally decreased. In humans, supplementation with oral NAC normalizes low levels of glutathione.

\section{Alpha-Lipoic Acid}

Recent studies have shown that when alpha-lipoic acid is added to different types of human or animal cells in culture, cellular glutathione levels increase by 30 $70 \%$. When daily doses of alpha-lipoic acid are injected daily into cells of lungs, livers or kidneys for 11 days, glutathione levels increase markedly.

\section{Whey Protein Concentrate}

Studies have shown that whey protein concentrate acts as a cysteine donor to increase the intracellular production of glutathione. In a study of 20 young healthy adult subjects, supplementation with whey protein concentrate resulted in a significant increase in lymphocyte glutathione over the placebo group. The researchers hypothesized that the increase in glutathione concentrations in peripheral blood lymphocytes resulted from regulation of glutathione production in response to oxidative stress and that whey protein concentrate acted effectively as a donor of cysteine. Subsequently, levels of blood glutathione increased significantly (94\%) after regular intake of whey protein concentrate by a patient 
treated for an obstructive upper track disease related to smoking, asthma and to lung cancer. In this case study, the researchers reported that the patient had shown a symptomatic improvement in lung function coinciding with the intake of milk protein concentrate. His condition had worsened when supplementation was interrupted and improved when milk protein concentrate had been reintroduced, leading researchers to make the following statement: Its respiratory status was objectively and subjectively better than in the four years above. In a study of HIV-infected patients, supplementation with whey protein concentrate significantly increased glutathione levels as more subjects were more likely to reach normal weight.

\section{Silymarin Increases Hepatic Glutathione Levels by $30 \%$}

The standardized extract of silymarin is a powerful antioxidant, widely used in Europe to treat liver diseases. It protects the liver from damage caused by alcohol absorption and, in particular, from decreased levels of glutathione in the liver. One month of supplementation with silymarin increases liver glutathione levels by $35 \%$.

\section{Same Protects the Liver's Antioxidant Defense System}

SAMe, S-adenosylmethionine, plays a particularly important role in the biochemistry of the liver. It is the product of a reaction between ATP and methionine. Nearly half of the methionine present in the body is used in the liver to produce SAMe. Much of the latter is itself transformed into glutathione.

The consumption of alcohol lowers the levels of an enzyme necessary for the transformation of methionine into SAMe. In 1991, a study by Spanish researchers confirmed the ability of SAMe to convert to glutathione. It has also shown that absorption of SAMe supplements helps maintain glutathione levels if they are absorbed at the same time as alcohol. The latter results in a sharp decrease in glutathione levels.

In a placebo-controlled study, 16 patients with alcoholic and non-alcoholic liver diseases received 1200 $\mathrm{mg}$ of SAMe daily for 6 months. Liver biopsies showed a significant increase in glutathione levels, along with decreased oxidized glutathione. In non-alcoholic subjects with liver damage, alanine aminotransferase, a liver enzyme indicative of lesions, was reduced.

The nanoantioxidant developed consists of a nanoparticle of silicon dioxide associated with gallic acid. The latter is one of the most effective antioxidants. However, it rapidly loses its effect, as the molecules of gallic acid tend to attach to each other, thus deactivating their actions. However, ETHZ researchers have succeeded in suppressing this phenomenon by associating the molecules of gallic acid with silicon dioxide. Because of its larger size than that of gallic acid, silicon dioxide prevents the molecules of gallic acid from interacting with each other. The nanoantioxidant thus formed possesses the remarkable antioxidant action of gallic acid and a prolonged life. It can thus be preserved more easily and be effective in smaller quantities. In addition, it is resistant to high temperatures, unlike conventional antioxidants.

\section{Results and Discussion}

Antioxidants are a group of compounds produced by the organism or that occur naturally in many foods. Antioxidants work together in the human body to maintain health and vigor, especially during the last decades of life (Rahman, 2007). They do this by protecting us from harm caused by free radicals that can destroy skin and tissues healthy cells of the organism. The body produces free radicals in the course of normal power production but there was substance in the environment that certain chemicals, smoke, contamination, solar radiation which stimulates free radical production. As a matter of fact free radicals are major guilty for the process of aging of the skin (Pandel et al., 2013) and of the organism, in general (Petrescu et al., 2015).

Through the process of checking the free radicals, the antioxidants can make a difference between life and death and can determine the speed and the way in which we age (Godic et al., 2014). Very many tests and scientific experiments demonstrate that the persons who are eating a diet rich in antioxidants and take a supplement of antioxidants, live longer and healthier (Schagen et al., 2012).

Corneum stratum (layer) of the skin is a barrier between the external and internal of the body. This explains why the stratum corneum thickens in places where the skin is subject to friction and pressure. The protective function of the skin is fulfilled provided that the skin is intact. Wrinkle is defined in DEX as - the cheek skin crease, wrinkle, wrinkles caused by tearing of the dermis elastic fibers. Skin wrinkling is the result of the following mechanisms: Aging, attendants thought the sequelae of actinic lesions and genetic disorders.

Visible morphological changes associated with the aging process are the following: Decrease bone structure, reduce skin thickness and elasticity to subadiacente layers, the gravitational fall of the soft tissue, the formation of wrinkles in areas of adhesion with deep structures and areas of muscle insertion.

There may be several categories of wrinkles:

- Wrinkles of expression -which may arise early, being related to face constrained, which prints a specific activity to the facial muscles

- Wrinkles due to aging which are divided into: Wrinkles of animation, resulting in contraction of mimic muscles; -fine wrinkles caused by breaking 
of the fibrillating network structure; -wrinkles pronounced produced by solar elastosis

The face makes wrinkles early due to facial skin mobility, driven by functions or mimic facial muscles. Age at which appear wrinkles, varies according to: The regime of life and habits, solar radiation, heat, cold, humidity, light too high or low, alcohol abuse, smoking, stress, diets, using cosmetics inappropriate, genetic baggage.

\section{Free Radicals Accelerate Aging}

Free Radicals meet many critical functions in our body, checking blood passing through artery up to the fight against infection. Some free radical signal molecules that is responsible for starting and stopping of genes. Some free radicals, such as nitric oxide and superoxide, are produced in large quantities by our cells immune to "poison" viruses and bacteria.

However in Less than a second, Free Radicals May Turn against us, we may Sicken and we can Grow Old too Fast

Where there is a stroke or a heart attack, or just an inflammatory disease such as arthritis, free radicals are existing or in production. For us to understand what are free radicals must be known human cells, where every day and every second, is wearing a war between free radicals and antioxidants.

As anything in the universe and the cells are made up from smaller units called atoms. Each atom contains a nucleus that is surrounded by electrons. Two or more atoms may be linked by dividing electrons forming molecules.

The biological process of oxidation (the process of training of energy), involve the transfer electrons from an oxygen molecule to the next. For these reasons sometimes may escape one lone electron.

An atom remained without one electron (an ion) present in human body, bears the name of a free radical.

Free radicals are formed constantly almost everywhere in the body in an amazing proportion. If free radicals spread by all over the body are not caught, may attack and oxidize DNA, genetic material that controls growth and development of cells, promote spiral down premature aging, or may cause many other heavy damage by intoxicating the body.

Many people consume network antioxidants-vitamin C, vitamin E, lipoic acid, coenzyme Q10, glutathione, etc because they want to look and feel younger. Keeping antioxidant use i.e., keeping free radicals in check can be one of the most effective ways to slow the aging process.

What became known as the free radical theory of aging was proposed in 1954 by Dr. Denham Harman, a researcher in Berkeley, studying the effects of radiation on human skin. In that time the United States was in the Cold War with the Soviet Union and there were fears that it will reach a nuclear war. Dr. Harman was commissioned by the then government to find an effective antidote to radiation poisoning resulting from an atomic attack.

What makes skin exposure to radiations to be so dangerous is that it attracts lethal hydroxyl radical production, the most powerful and extremely dangerous free radical known. This free radical usually occurs when water comes into contact with ionizing radiation.

Hydroxyl radical is highly reactive, destroying everything in its path. Once created it is almost impossible to be stopped. Dr. Harman was the first to make the connection between free radicals produced during irradiation and free radicals produced by normal production of energy in the human body.

Radiation poisoning produced symptoms comparable with aging and hypothesized idea that free radicals are responsible for producing the same effect but in a longer period of time.

It was long believed that the key to revealing the mysteries of aging lies in human cells-(mode in how cells use oxygen to produce energy).

Dr. Harman has shown that bio-oxidation product of free radicals from human body, essentially contributes to the aging process and while we can't prevent the passing years or aging body, but we can use antioxidants to reduce damage caused by free radicals, thus slowing down the aging process.

Free radicals aging both of body: Outside and inside. Ultraviolet radiation from the sun, when they touch the skin excites a molecule on the surface of the skin, which reacts with oxygen to form singlet (atomic) oxygen. The atomic oxygen generates the production of free radicals including ozone.

What actually happens? Ultraviolet radiations are those who manage to split water into oxygen and hydrogen. This can be used in the automotive industry but it is not useful to the human body. Ultraviolet radiations manage to break the water present on the skin but also in tissues.

The human body is composed of $70 \%$ water, but any uncontrolled loss can disrupt the human machine. In addition atomic oxygen produced quickly turn into ozone, an extremely toxic to the human body. On how atmospheric ozone is necessary to clean the air of toxic byproducts and stop and limit the penetration of ultraviolet radiation in the Earth's atmosphere as it is toxic and dangerous to the earth's surface or in the human body. Aging skin tissue occurs through dehydration and by the attack of the free radicals produced including the resulting ozone.

Ozone is an unstable compound consisting of three oxygen atoms bound together, in which only one normal and the other two are ions, one positive and the other negative.

While it is not a free radical ozone promotes the formation of free radicals. Ozone not only attacks the skin, it can destroy the lining of lungs, mouth and nasal cavity. 
And in free radicals produced by solar radiation with dehydration and in those produced in the human body hydration is a serious and useful remedy immediately (because water has a cleansing effect and antioxidant). But to combat permanent free radicals inside our and their negative effects on the human body, requires a permanent cure with network antioxidants.

\section{The Network Antioxidants}

Antioxidants network (network of special antioxidants) work together in the body (Packer and Colman, 1999). Only five are considered the network antioxidants: Vitamin $\mathrm{C}$, Vitamin E, lipoic acid, coenzyme Q10, glutathione. Vitamin $\mathrm{C}$ and $\mathrm{E}$ are not produced by our body but must be obtained through food.

Lipoic acid, CoQ10 and glutathione are produced by our body, but levels of these antioxidants decreases as we age. Scientists worldwide are investigating the role of antioxidants in preventing skin aging and disease prevention in aging.

Many people think that inherit genes premature aging or inherit a tendency to develop skin cancer. These five antioxidants can stop bad genes and reduce the risk of developing hereditary diseases. Antioxidants may delete premature brown spots and other signs of skin degraded by sunlight. Antioxidants may erase the signs of aging skin and protect the skin cancer (Ganceviciene et al., 2012).

Parker Laboratory (Packer and Colman, 1999) of the University of California-Berkeley include an antioxidant skin care regimen that not only prevent skin cancer but can slow down and even eliminate wrinkles, fine lines and other signs of aging skin.

Antioxidants are the body police against free radicals, call whenever needed to quell free radicals where they are so that their destructive power can't be extended to other cells.

When an antioxidant encounters a free radical, the radical is automatically joined to antioxidant molecular structure. Antioxidant becomes a new type of free radical, harmless. This will exempt cells and tissue destructive action of a radically freely got out of control.

What really makes the network of antioxidants to be special is that they can "recycle" or regenerate after they were loosened a radically free, widening and more power.

For Example: When Vitamin C Triggers a radically free it becomes Weak free Radical in the Process

This anti-oxidant can be recycled in the form of vitamin C. As an anti-oxidant network saves him on the other side, cycle continues by making sure that the body will keep the correct balance of the antioxidants.

This particular scenario-the antioxidant meets a free radical-it take it, becomes a free radical friend-is recycled for another antioxidant network-occurs repeatedly in the body, in the blink of an eye.
Research has estimated that the number knocks oxidative degradation administered daily DNA in each cell is 10,000 and if this figure is multiplied by the millions of cells in the body can be understood the scale of such processes in the human body.

Each antioxidant has a unique niche in the cell; for example in the areas of aqueous cell or in the blood, which consists primarily of water, they will act only vitamin $\mathrm{C}$ and glutathione.

\section{The Role of Vitamins $C$ and $E$ in Maintaining Young Skin}

Vitamin C prevents skin cancer, guarding DNA of threat free radicals. In normal amounts Vitamin $\mathrm{C}$ provides beautiful skin (Marx et al., 2000). Vitamin C is essential for collagen production cellular glue that holds the body bound and keep skin young and supple. Vitamin $\mathrm{C}$ protects the body against health problems especially in the elderly. Vitamin $\mathrm{C}$ is considered the center of the antioxidant network, because the connection between fat-soluble antioxidants and the water soluble (Bojovic and Stojanovic, 2005).

Vitamin C is Important for Reloading a Fat-Soluble Vitamin E; when it becomes a free Radical, it best Meets Function

Vitamin C (ascorbic acid) is found in the following foods: Citrus, green peppers, broccoli, blueberries, strawberries, tomatoes, cabbage. The daily requirement of Vitamin C: $90 \mathrm{mg}$ /day for men, $75 \mathrm{mg}$ /day women, 100 mg/day smokers (Pérez-Ruíz et al., 1995).

Glutathione -is the main water-soluble antioxidant. In the network of antioxidants, glutathione recycles the oxidative form of vitamin $\mathrm{C}$, restoring its oxidative power. Product of the body contributes to liver detoxification of pollutants and drugs. Glutathione is important for a strong immune system; an increase in the levels of glutathione can redress the fall due to the age of the immune function. Glutathione is found in fruits, vegetables, fresh meat cooked (Pisoschi et al., 2011).

Vitamin $\mathrm{E}$ is an antioxidant against the aging process; reverses the fall of immune function with age and helps brain cells to do not become old. Vitamin E keeps the skin young and protects from UV radiation and ozone, especially against skin cancer.

In the Network of Antioxidants Vitamin $E$ is Recycled by Vitamin C, Lipoic acid and Coenzyme Q10

Vitamin E can flow through the thick portions of the cell membrane searching and eliminating free radicals.

Vitamin E can be found in the following foods: Raw vegetable oil, butter walnut, rice oil, nuts, rice, green vegetables with leaves, broccoli, carrot, mango, zucchini, papaya. The required daily dose for an individual is about $30 \mathrm{mg} /$ day. 


\section{Antioxidant Miracle-slowing Down the Aging Process}

Strategic use of antioxidant network as suggested plan Packer can stop and even solve problems associated with aging. Today though some people live longer than ever, achieving the age of 120 years associated with life expectancy is a rarity.

Theory free radicals which cause aging, the alleged for the first time by the laboratories Packer declares that aging is a cumulative damage of cells and tissues produced for several decades due to exposure to free radicals.

\section{In Nature there is a Direct Connection between} High Level of Antioxidants and the Life Expectancy

The protective effect of antioxidants is striking if we try to correlate the average lifetime of different species of mammals with their level of antioxidants. In each case, the species with the highest amount of vitamin E or the strongest antioxidant defense system live most.

Humans and elephants are in the forefront, both in terms of antioxidant activity and in life expectancy, whereas mice and other rodents (with antioxidant least) living low.

Numerous studies have shown that antioxidants can prevent many of the signs of aging of the cells announced. Thus, in a culture of human cells by adding the vitamin $\mathrm{E}$ has doubled the life of human cells.

Antioxidants can prevent signs of aging announced by cell, namely the accumulation of aging pigment called lipofuscin, in all specialized cells of the body, especially in the brain.

Lipofuscin is a direct result of lipid oxidation, accelerating the aging process in the tube-like body. Antioxidants may greatly slow down the formation of lipofuscin, keeping young cells for proper a longer period of time.

Antioxidants can prevent the formation of AGEs (advanced glycation end-products). Consumption of refined sugar triggers a process called glycation (glicosilation non-enzymatic) by which sugar molecules attach to proteins.

This results in novel proteins referred to as harmful according to the "AGE" (Advanced Glycation Endproducts, end products of advanced glycation), which damage the cell and collagen proteins are more rigid with decreasing elasticity. Glycation occurs in all cells of the body and is accelerated by a diet rich in sugars as well as prolonged exposure to sunlight.

Glycation occurs when glucose reacts with proteins, resulting in binding of the protein chain and the formation of free radicals. Too many of these proteins can lead to external signs of premature aging such as wrinkles and brown spots on the skin and cause even more damage inside the body (Fig. 1) (Rimbach et al., 2005).

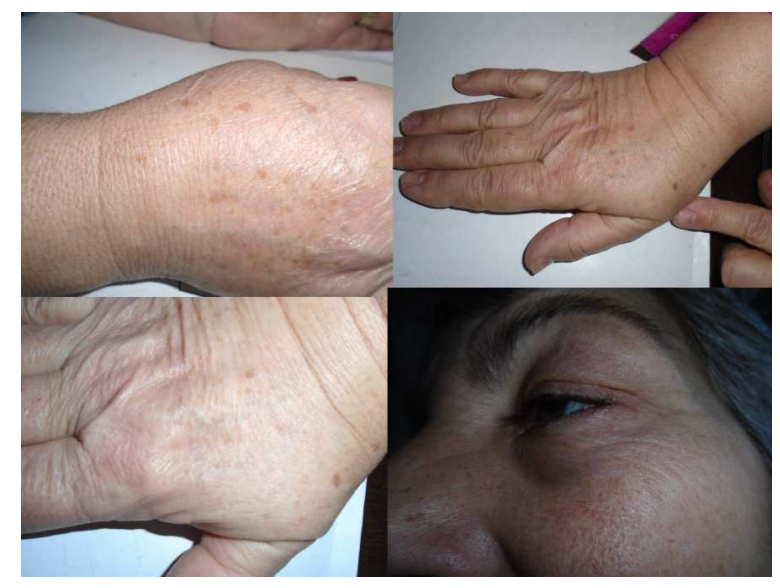

Fig. 1. External signs of premature aging such as wrinkles and brown spots on the skin

\section{Conclusion}

The development of modern methods and fast like this still takes place with or without the consent of many existing opposing forces on the planet today who do not want the development of mankind and in this way simply because they follow other interest groups (usually financial).

Any important contribution to people's health and extend life with health is a serious scientific contribution and deserve to be taken into account.

The best way to prolong life is to strengthen the antioxidant defense network that can control the forces that drive the aging process.

The main antioxidants that must be consumed daily (especially that can't be synthesized by the human body) are vitamins $\mathrm{C}$ and $\mathrm{E}$.

A daily moisturizing wider also has a key role in active protection of the body against free radicals internal and external.

A well thought permanent food diet can help essentially to balance and strengthen the immune system of our body.

The best way to prolong life is to strengthen the antioxidant defense network that can control the forces that drive the aging process. The main antioxidants that must be consumed daily are vitamins $\mathrm{C}, \mathrm{E}$ and $\mathrm{A}$. This is done easiest by a diet rich in fruits, vegetables and fresh greens.

Antioxidants can prevent signs of aging announced by cell, namely the accumulation of aging pigment called lipofuscin, in all specialized cells of the body, especially in the brain.

\section{Acknowledgement}

This text was acknowledged and appreciated by Assoc. Pro. Taher M. Abu-Lebdeh, North Carolina A 
and T State Univesity, United States, Muftah H. ElNaas PhD MCIC FICCE QAFCO Chair Professor in Chemical Process Engineering Gas Processing Center College of Engineering Qatar University, Professor Guanying Chen Harbin Institute of Technology and SUNY Buffalo China and (Ms.) Shweta Agarwala Senior Research Scientist at Singapore Center for 3D Printing Nanyang Technological University Singapore, whom we thanks and in this way.

\section{Author's Contributions}

All the authors contributed equally to prepare, develop and carry out this manuscript.

\section{Ethics}

This article is original and contains unpublished material. The corresponding author confirms that all of the other authors have read and approved the manuscript and no ethical issues involved.

\section{References}

Ahmed, A. and T. Tollefsbol, 2001. Telomeres and telomerase: Basic science implications for aging. J. Am. Geriatr Soc., 49: 1105-1109.

DOI: 10.1046/j.1532-5415.2001.49217.x

Alexeyev, M.F., S.P. LeDoux and G.L. Wilson, 2004. Mitochondrial DNA and aging. Clin. Sci., 107: 355-364. DOI: $10.1042 / \mathrm{CS} 20040148$

Allen, J.A. and M.M. Coombs, 1980. Covalent binding of polycyclic aromatic compounds to mitochondrial and nuclear DNA. Nature, 287: 244-245.

DOI: $10.1038 / 287244 \mathrm{a} 0$

Ames, B.N., 1989. Endogenous oxidative DNA damage, aging and cancer. Free Radic. Res. Commun., 7: 121-128. DOI: 10.3109/10715768909087933

Anisimov, V.N., 2001. Life span extension and cancer risk: Myths and reality. Exp. Gerontol., 36: 1101-1136. DOI: 10.1016/S0531-5565(01)00114-0

Artandi, S.E., 2006. Telomeres, telomerase and human disease. N Engl. J. Med., 355: 1195-1197. DOI: $10.1056 / \mathrm{NEJMp} 068187$

Austad, S.N., 1993. Retarded senescence in an insular population of Virginia opossums (Didelphis virginiana). J. Zool., 229: 695-708. DOI: 10.1111/j.1469-7998.1993.tb02665.x

Beckman, K.B. and B.N. Ames, 1998. The free radical theory of aging matures. Physiol. Rev., 78: 547-81. PMID: 9562038

Besedovsky, H. and R.A. Del, 2008. Immune-neuroendocrine interactions: Facts and hypotheses. Endocrinol. Rev., 17: 1945-7189.

DOI: 10.1210/edrv-17-1-64\#sthash.SRXTG0Xy.dpuf
Blackburn, E.H., 2000. Telomere states and cell fates. Nature, 408: 53-56. DOI: 10.1038/35040500

Bojovic, B. and J. Stojanovic, 2005. Chlorophyll and Carotenoid Content in wheat cultivars as a function of mineral nutrition, Arch. Biol. Sci., 57: 283-290. DOI: $10.2298 / \mathrm{ABS} 0504283 \mathrm{~B}$

Cadenas, E. and K.J. Davies, 2000. Mitochondrial free radical generation, oxidative stress and aging. Free Radic. Biol. Med., 29: 222-230. DOI: $10.1016 / \mathrm{S} 0891-5849(00) 00317-8$

Campisi, J., 1997. The biology of replicative senescence. Eur. J. Cancer, 33: 703-709. DOI: $10.1016 / \mathrm{S} 0959-8049(96) 00058-5$

Cong, Y.S., J. Wen and S. Bacchetti, 1999. The human telomerase catalytic subunit htert: Organization of the gene and characterization of the promoter. Hum. Mol. Genet., 8: 137-142. DOI: 10.1093/hmg/8.1.137

Daynes, R.A. and B.A. Araneo, 1992. Prevention and reversal of some age-associated changes in immunologic responses by supplemental dehydroepiandrosterone sulfate therapy. Aging: Immunol. Inf. Dis., 3: 135-153.

De, L. and M. Fuente, 2002. Effects of antioxidants on immune system ageing. Eur. J. Clin. Nutr., 56: S5-S8. DOI: 10.1038/sj.ejcn.1601476

Deligiannakis, Y., G.A. Sotiriou and S.E. Pratsinis, 2012. Antioxidant and antiradical $\mathrm{SiO}_{2}$ nanoparticles covalently functionalized with gallic acid. ACS Appl. Mat. Int., 4: 6609-6617.

DOI: $10.1021 / \mathrm{am} 301751 \mathrm{~s}$

Dirks, A.J. and C. Leeuwenburgh, 2006. Caloric restriction in humans: Potential pitfalls and health concerns. Mech. Age. Dev., 127: 1-7. DOI: 10.1016/j.mad.2005.09.001

Fabris, N., 1991. Neuroendocrine-immune interactions: A theoretical approach to ageing. Arch. Gerontol. Geriatr., 12: 219-230. DOI: 10.1016/0167-4943(91)90029-P

Finkel, T. and N.J. Holbrook, 2000. Oxidants, oxidative stress and the biology of ageing. Nature, 408: 239-247. DOI: $10.1038 / 35041687$

Foy, C.J., A.P. Passmore, M.D. Vahidassr, I.S. Young and J.T. Lawson, 1999. Plasma chain-breaking antioxidants in Alzheimer's disease, vascular dementia and Parkinson's disease. QJM, 92: 39-45. DOI: 10.1093/qjmed/92.1.39

Franceschi, C., 1989. Cell proliferation and cell death in the aging process. Ag. Clin. Exp. Res., 1: 3-15. DOI: $10.1007 / \mathrm{BF} 03323871$

Ganceviciene, R., A.I. Liakou, A. Theodoridis, E. Makrantonaki and C.C. Zouboulis. 2012. Skin anti-aging strategies. Dermato Endocrinol., 4: 308-319. DOI: 10.4161/derm. 22804

Godic, A., B. Poljšak, M. Adamic and R. Dahmane, 2014. The role of antioxidants in skin cancer prevention and treatment. Oxidat. Med. Cellul. Longev., 2014: 860479-860484. DOI: $10.1155 / 2014 / 860479$ 
Harman, D., 2003. The free radical theory of aging. Antioxid Redox. Signal., 5: 557-561. DOI: $10.1089 / 152308603770310202$

Harman, D., 1956. Aging: A theory based on free radical and radiation chemistry. J. Gerontol., 11: 298-300. DOI: $10.1093 /$ geronj/11.3.298

Harman, D., 1962. Role of free radicals in mutation, cancer, aging and the maintenance of life. Radiat. Res., 16: 753-763. DOI: $10.2307 / 3571274$

Harman, D., 1969. Prolongation of life: Rote of free radical reactions in aging. J. Am. Geriatr. Soc., 17: 721-735. DOI: 10.1111/j.1532-5415.1969.tb02286.x

Harman, D., 1972. The biologic clock: The mitochondria. J. Am. Geriatr. Soc., 20: 145-147. DOI: $10.1111 /$ j.1532-5415.1972.tb00787.x

Harman, D., 1981. The aging process. Proc. Natl. Acad. Sci. USA, 78: 7124-7128.

Hayflick, L., 2004. Anti-aging is an oxymoron. J. Gerontol A Biol. Sci. Med. Sci., 59A: 573-578. DOI: $10.1093 /$ gerona/59.6.B573

Holliday, R., 2006. Aging is no longer an unsolved problem in biology. Ann. N Y Acad Sci., 1067: 1-9. DOI: $10.1196 /$ annals. 1354.002

Huerre, M.R. and P. Gounon, 1996. Inflammation: Patterns and new concepts. Res. Immunol., 147: 417-434. DOI: 10.1016/S0923-2494(97)84407-0

Kang, J.H., N. Cook, J. Manson, J.E. Buring and C.M. Albert et al., 2009. Vitamin E, Vitamin C, beta carotene and cognitive function among women with or at risk of cardiovascular disease. Women's Antiox. Cardiovascular Study, 119: 2772-2780. DOI: 10.1161/CIRCULATIONAHA.108.816900

Kanungo, M.S., 1975. A model for ageing. J. Theor Biol., 53: 253-261.

DOI: $10.1016 / \mathrm{S} 0022-5193(75) 80002-6$

Knekt, P., A. Reunanen, A. Aromaa, M. Heliövaara and T. Hakulinen et al., 1988. Serum cholesterol and risk of cancer in a cohort of 39,000 men and women. J. Clin. Epidemiol., 41: 519-530. DOI: $10.1016 / 0895-4356(88) 90056-X$

Knight, J.A., 2000. Review: Free radicals, antioxidants and immune system. Ann. Clin. Lab Sci., 30: 145-158. PMID: 10807157

Kowald, A. and T.B. Kirkwood, 1996. A network theory of ageing: The interactions of defective mitochondria, aberrant proteins, free radicals and scavengers in the ageing process. Mutat. Res., 316: 209-236. DOI: 10.1016/S0921-8734(96)90005-3

La Fata, F.G., P. Weber and M.H. Mohajeri, 2014. Effects of vitamin $\mathrm{E}$ on cognitive performance during ageing and in alzheimer's disease. Nutrients, 6: 5453-5472. DOI: 10.3390/nu6125453

Marx, M., A. Schieber and R. Carle, 2000. Quantitative determination of carotene stereoisomers in carrot juices and vitamin supplemented (ATBC) drinks. Food Chemist, 70: 403-408.

DOI: $10.1016 / \mathrm{S} 0308-8146(00) 00096-0$
Mccall, M.R. and B. Frei, 1999. Can antioxidant vitamins materially reduce oxidative damage in humans. Free Radic. Biol. Med., 26: 1034-1053. DOI: 10.1016/S0891-5849(98)00302-5

Meydani, S.N. and J.B. Blumberg, 1989. Nutrition and the Immune Function in the Elderly. In: Human Nutrition: A Comprehensive Treatise, Munro, H. and A. Danforth (Eds.), Plenum Press, New York, pp: 61-87.

Morris, M.C., 2009. The role of nutrition in Alzheimer's disease: Epidemiological evidence. Eur. J. Neurol., 16: 1-7. DOI: 10.1111/j.1468-1331.2009.02735.x

Packer, L and C. Colman, 1999. The Antioxidant Miracle-Put Lipoic Acid, Pycnogenal and Vitatamins $\mathrm{E}$ and $\mathrm{C}$ to Work for you. 1st Edn., Wiley, ISBN-10: 0471353116. pp: 256.

Pandel, R., B. Poljšak, A. Godic and R. Dahmane, 2013. Skin Photoaging and the role of antioxidants in its prevention. ISRN Dermatol., 2013: 11-12. DOI: $10.1155 / 2013 / 930164$

Paolisso, G., M.R. Tagliamonte, M.R. Rizzo, D. Manzella and A. Gambardella et al., 1998. Oxidative stress and advancing age: Results in healthy centenarians. J. Am. Geriatr Soc., 46: 833-838. DOI: $10.1111 /$ j.1532-5415.1998.tb02716.x

Pérez-Ruíz, T., C. Martínez-Lozano and A. Sanz, 1995. Flow-injection chemiluminometric determination of ascorbic acid based on its sensitized photooxidation. Analytica Chimica Acta., 308: 299-307. DOI: 10.1016/0003-2670(94)00527-S

Petrescu, F.L., E. Buzea, L. Nănuţ, M. Neacşa and C. Nan, 2015. The role of antioxidants in slowing aging of skin in a human. Analele Universitatii Din Craiova Biol. Horticul. Tehnol. Prelucrarii Produsel. Agricole Ingineria Mediului., 20: 567-574. DOI:

Pisoschi, A.M., A. Pop, G.H.P. Negulescu and A. Pisoschi, 2011. Determination of ascorbic acid content of some fruit juices and wine by voltammetry performed at $\mathrm{Pt}$ and carbon paste electrodes. Molecules, 16: 1349-1365. DOI: $10.3390 /$ molecules16021349

Rahman, K., 2007. Studies on free radicals, antioxidants and co-factors. Clin. Int. Ag., 2: 219-236. PMID: 5804024

Ravindran, R.D., P. Vashist and S.K. Gupta, 2011. Inverse association of vitamin $\mathrm{c}$ with cataract in older people in india. Ophthalmol, 118: 1958-1965. DOI: $10.1016 /$ j.ophtha.2011.03.016

Richter, C., J.W. Park and B.N. Ames, 1988. Normal oxidative damage to mitochondrial and nuclear DNA is extensive. Proc. Natl Acad Sci., 85: 6465-6467.

Rimbach, G.., L. Packer and J. Fuchs, 2005. Nutrigenomics (Oxidativ stress and disease). Pediatric Endocrinol. Rev. 
Rimm, E.B., M.J. Stampfer, A. Ascherio, E. Giovannucci and G.A. Colditz et al., 1993. Vitamin E consumption and the risk of coronary heart disease in men. N Engl. J. Med., 328: 1450-1456. DOI: 10.1056/NEJM199305203282004

Satoh, J., N. Kohara, K. Sekiguchi and Y. Yamaguchi, 2016. Effect of ranirestat on sensory and motor nerve function in japanese patients with diabetic polyneuropathy: A randomized double-blind placebo-controlled study. J. Diabet. Res., 2016: 5383797-5383804. DOI: 10.1155/2016/5383797

Satoh, J., N. Kohara and C. Hamada, 2013. Assessment of the reliability of a Japanese version of the modified toronto clinical neuropathy score in Japanese patients with diabetic sensorimotor polyneuropathy. J. Japan Diabet. Society, 56: 932-937. DOI: $10.1111 /$ j.2040-1124.2010.00070.x

Schagen, S.K., V.A. Zampeli, E. Makrantonaki and C.C. Zouboulis, 2012. Discovering the link between nutrition and skin aging. Dermato Endocrinol., 4: 298-307. DOI: 10.4161/derm. 22876
Stephens, N.G., 1996. Randomised controlled trial of vitamin $\mathrm{E}$ in patients with coronary disease: Cambridge Heart Antioxidant Study (CHAOS). Lancet, 347: 781-786.

Tosato, M., V. Zamboni, A. Ferrini and M. Cesari, 2007. The aging process and potential interventions to extend life expectancy. Clin. Int. Ag., 2: 401-412 PMID: 18044191

Viña, J., C. Borras, K.M. Abdelaziz, R. Garcia-Valles and M.C. Gomez-Cabrera, 2013. The free radical theory of aging revisited: The cell signaling disruption theory of aging. Antiox. Redox. Signal., 19:779-787. DOI: 10.1089/ars.2012.5111

Wick, M., D. Zubov and G. Hagen, 1999. Genomic organization and promoter characterization of the gene encoding the human telomerase reverse transcriptase (hTERT). Gene, 232: 97-106. DOI: 10.1016/S0378-1119(99)00108-0 